\title{
Dark Matter Frequency in Complex Space
}

\author{
J.A.de Wet \\ PO Box 514, Plettenberg Bay, 6600, South Africa
}

Abstract. In this note we propose that Dark Matter belongs to the complex space of the exceptional lie Algebra E6.This is why it has not been observed, but does have a fundamental frequency $f$.

Key Words: E6, Equiharmonic Lattice, Quarks, Hessian Polyhedron.

\section{Introduction}

In a recent paper on 'What are Quarks' [2] it was proposed that quarks belong to the imaginary axis of the graph of the Hessian Polyhedron E6 with 3 real and 3 imaginary axes. This is depicted in Fig.1 below, where the imaginary vertices representing the 3 up and down quarks u,d that make up protons and neutrons and their anti-particles are in the outer circle; leaving the inner ring for leptons and strange particles with the heavy $\tau^{+-}$and $v_{\tau}$ particles in the center. Fig. 1 is actually a projection $\mathrm{E}_{6} / \mathrm{F}_{4}$ and is the subalgebra $\left(\mathrm{su}_{3}\right)_{\text {color }} \mathrm{x}\left(\mathrm{su}_{3}\right)_{\operatorname{spin}} \mathrm{x}$ $\left(\mathrm{su}_{3}\right)_{\text {isospin }}$. This figure was authenticated by the employment of Theta Functions which are essentially a rotation in a space with one real co-ordinate $\mathrm{K}$ and one complex co-ordinate $\mathrm{iK}$ which are linked by the exponential nome $\mathrm{q}$ of the ratio $\frac{i K}{K}$ that are quarter periods on the real and imaginary axes.Particularly if these are equiharmonic, or multiples of a commonfrequency f. Then $\mathrm{q}=0.06583=\exp \frac{\pi i K}{K}$ or $\frac{i K}{K}=\frac{\sqrt{3}}{2}=\sin 120^{\circ}$ or $\sin 60^{\circ}$ (1)

Which is precisely the angle in Fig. 1 that defines quarks and anti-quarks in an equiharmonic lattice [3,Ch.4]. In this way the E6 lattice of Fig. 1 defines a coupling constant uniting up and down quarks and the fundamental frequency $f$ could be electromagnetic in 3space or Dark Matter frequency in complex space which, according to Arvanitaki [1] of the Perimeter Institute, may be almost audible resonating in the kilohertz range. If Dark Matter is in complex spaceCP ${ }^{3}$ then this would explain why it is not seen.

\section{References}

[1]. Arvanitaki, The Dark Matter signal, Weblog /2016/ 03. Online

[2]. J.A.de Wet, What are Quarks? IOSR-JM , Vol. 13 (2017)

[3]. Bruce Hunt, The Geometry of some Special Arithmetic Constants, Lecture Notes in Mathematics, Springer (1637) 


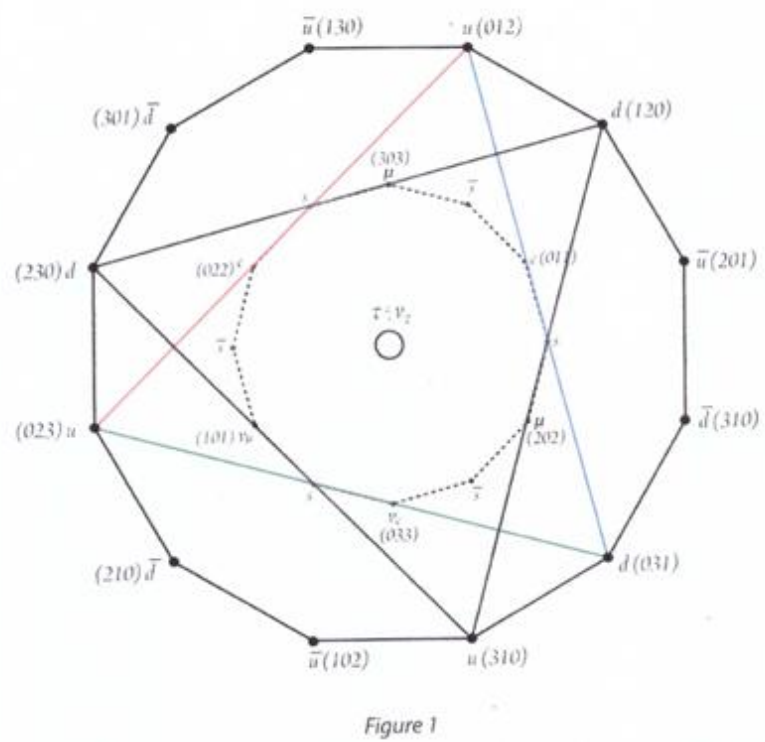

Figure Captions

Figure 1. Caption of figur 